$B U-303-M$

\title{
NONVERBAL INSTRUCTIONAL APPROACHES FOR \\ INTRODUCTORY STATISTICS
}

by

N. S. Urquhart

ABSTRACT

The intent of this paper is to identify some of the common difficulties of introductory courses, and to use an introductory statistics course as a vehicle for illustrating specific solutions to these problems. The term "nonverbal instructional approach" is intended to convey the idea of an instructional approach which contains a substantial component which is nonverbal; it rarely would be used devoid of words. Computers, models and pictures serve as illustrations from statistics.

Paper No. BU-182 in the Biometrics Unit Series and No. 574 in the Department of Plant Breeding and Biometry Series, Cornell University, Ithaca, New York 14850. 
NONVERBAL INTSTRUTTIONNAI APPROACHES FRR

INTRODUCTORY STATISTICS

by

N. S. Urquhart*

The students in any course constitute a rich mosaic of many attitudes, prejudices, fears, interests and preconceived notions. This should have many implications on our instructional behavior, but it seems to be especially relevant to the teaching of introductory courses. The intent of this paper is to identify some of the common difficulties of introductory courses, and to use an introductory statistics course as a vehicle for illustrating specific solutions to these problems. The term "nonverbal instructional approach" is intended to convey the idea of an instructional approach which contains a substantial component which is nonverbal; it rarely would be used devoid of words. Computers, models and pictures serve as illustrations from statistics. Puzzles, games, and laboratory exercises, field trips and experiments are examples which could be used in other areas.

\section{INTRODUCTORY COURSES}

Introductory courses are offered at all levels and in all subject-matter areas; they are taught for both major students and non-majors. The "service courses" taught for non-majors frequently suffer from such severe departmental disinterest that their teaching may be reserved for the faculty member who can teach nothing else or for the youngest and least experienced instructor, although there are notable exceptions to this. The objectives of an introductory course may even be ill-defined; but if it is to be introductory, it certainly must homogenize an otherwise heterogeneous group of students with regard to some aspect of the subject under consideration.

The student composition of an introductory course poses a problem at least as severe as any of the external difficulties. Students are much more diverse

* Assistant Professor of Biological Statistics, Biometrics Unit, Cornell University, Ithaca, New York 14850. 
than the automobiles on our highways. Yet, the mechanic trying to determine the ills of an automobile often must know something of its history as well as its observable characteristics before he can make an accurate diagnosis. The same is true of students; they are heterogeneous in many ways, and in the teaching of an introductory course, we would be remiss to ignore their diversity with regard to motivation, previous preparation and disciplinary interests.

Students have various reasons for being in an introductory course, particularly a course for non-majors. It may be required by their major curriculum. Even if the relevance of the course is apparent, attitudes will range from high motivation to outright disinterest, from enthusiasm to fright; if its relevance is questioned, attitudes will range from tolerance to hostility. A student who elects an introductory course is more apt to approach it with a positive attitude, but he may still be apprehensive about it if he elected it because he thought he needed it. If we fail to acknowledge this motivational heterogeneity, particularly at the beginning, we will fail to communicate with a significant portion of our students who nevertheless are entirely capable of mastering the subject.

An introductory course will be far more of an introduction, i.e., an initial familiarization, for some students than for others. Some encounter related material in high school, in other courses (related or apparently unrelated), by independent study and from everyday experience in work and travel. To ignore this is merely to give students another reason to be alienated toward the course; to accommodate it in our teaching is to give us a better chance of reaching the students, but at the expense of a more difficult teaching job.

In general, an introductory course for non-majors will have students with differing disciplinary interests. The extent of heterogeneity along this axis will depend somewhat upon the course. For example, an introduction to humanities, social science, psychology, etc. will cut across almost all disciplines; an introduction to chemistry will be populated by fewer disciplines while an introduction to microbiology will be yet more limited. A common way to eliminate such heterogeneity is by prerequisites and/or sectioning. Carried to its extreme, this would result in many sections with few students each---ideally with only one student in each section. Until either the supply of instructors increases or high quality student-computer Interaction is possible, this utopia is unattainable. In fact, there is some question as to whether it is utopian. Interaction among students 
produces a significant portion of their learning; some variability in attitudes, ability, etc. certainly enhances this process. There is the further question as to whether effective prerequisites or sectioning can be developed, especially in the case of an introductory course. Thus, it seems apparent that the large introductory courses taught to a relatively heterogeneous class are not going to die out soon; this is especially true in the large universities.

Given the responsibility for teaching an introductory course, how might you change it to accommodate the difficulties sketched above? You can change its objectives or its teaching approach, or better yet, both. Again, the objectives of an introductory course are to homogenize an otherwise heterogeneous group of students with regard to some aspect of the subject under consideration. The choice of the aspect to be homogenized is critical. An introduction, particularly for the non-major, should provide a synthesis of the basic approaches, concepts and schools of thought in the subject rather than isolated, perhaps technical, topics which would produce limited insight into the approaches of the discipline. On the other hand, there is practically unlimited opportunity for developing new approaches to the teaching of the subject if you are prepared to sit back and give serious consideration to it.

The gifted lecturer communicates hls central point by skillfully painting each of its parts and helping his audience view them from the proper perspective. His listeners can then put the whole picture together like the pieces of a jigsaw puzzle to see it for themselves. We might call this an indirect or inductive instructional approach in contrast to the familiar direct transmission of information through lectures and assigned readings. The use of the indirect approach is not limited to the gifted lecturer; 1t receives extensive use through the medium of laboratory experiences in the natural sciences. Hence the value of the indirect approach is widely accepted, a.t least tacitly. It is a pity that such approaches are used sparingly in lectures and almost never in "theoretical" subjects such as mathematics. Many indirect approaches might be characterized as being "nonverbal" in the sense that they involve doing, seeing, or experiencing rather than hearing or reading. Some of these can be used successfully in the lecture halls by less than gifted lecturers. The plea here is that we recognize nonverbal approaches as one powerful tool we should use to introduce and explain important concepts. 
In short, an introductory course needs to be taught with a realization that it contains students with diverse motivations and anxieties with regard to the course; it must contain material which will challenge the best-prepared student without losing the less well-prepared student; it must relate to the student's interest, especially in our present situation where students are so concerned about relevance. These difficulties can be tackled by altering the objectives of the course or its teaching approaches or both. Both were examined and modified for the introductory statistics course which is discussed in the sequel.

\section{EXAMPIE: AN INTRODUCTORY STATISTICS COURSE}

The statistics course used here as an example is taught as a part of the course offerings in the New York State College of Agriculture at Cornell University. The objectives and extent of student activity are somewhat influenced by the fact that it is taught mainly, but not exclusively, for graduate students in the life sciences and related social sciences. The class of about 175 students is taught by one professor, assisted by four graduate students in statistics. Since rational section schemes produce 15 to 20 sections, it was decided to have one class, but to seek ways to accommodate its heterogeneity and capitalize upon its size. The class now meets together three times a week for lectures and breaks up into smaller groups for laboratories (20 students) and discussion groups (10 students). If this course were to be mainly for undergraduates, then either some of the topics should be abbreviated or the presentation should be extended over more time, i.e., more credit hours or more semesters. However, the issues and approaches discussed here are independent of the level at which the course is taught.

Statistics is the science and art of obtaining, analyzing and interpreting data for the purpose of reaching conclusions; it finds application in all areas which generalize from experimental or observational data. As a science it is a mathematically rigorous subject which deals with the description, comparison and relation of populations; often in terms of parameters which characterize certain attributes of the populations. These mathematical considerations are based on certain assumptions which can rarely, if ever, be validated in the real world. Consequently, the art of statistics seeks to increase the objectivity with which we regard experimental or observational data for the purpose of describing, com- 
paring or relating populations about which our assumptions are questionable. That is, the art of statistics is a synthesis of the science of statistics with principles af experimentation, educated guesses about assumptions, and the folklore and experience from many experimental areas. Once data is available, the process of applying statistics to real world problems generally involves several steps: translation of the experimental concern into a statistical concern, (2) selection of a statistical procedure which is responsive to the statistical concern, (3) application of the statistical procedure to the available data, (4) determination of statistical conclusions, (5) translation of the statistical conclusions into experimental conclusions.

An introductory statistics course could thus be addressed to many concerns. It could, for example, consider mathematical statistics and seek to teach the development of statistical techniques within a given assumption framework. or it could teach the application of specified statistical techniques to available data. without considering the origin of the techniques. This corresponds to steps (3) and (4) enumerated in the preceding paragraph. Introductory statistical methods courses purport to teach the application of statistics to real world problems, but if available textbooks are any gauge to what is presently taught in these courses, they teach very little more than the application of specified statistical techniques to available data.

A course can have any set of objectives, but there is room for concern if its actual and intended objectives do not agree. In the present case, the intent is to teach students enough about statistics that they can intelligently apply it to problems of interest to them. This means that they must learn something about the translation - selection - application - conclusion - translation process as it is applied to various aspects of their several areas of subject-matter interest. Since these are activity oriented goals, the teaching of them should involve students in these or closely related activities. The goal of teaching students how to select an appropriate technique is the most difficult to achieve because it cannot be practiced until late in the term after they have encountered several techniques among which they can select. The translation goals require that considerable attention be given to concepts which are usually discussed only in mathematical statistics courses. These concepts must be developed without resorting to mathematical arguments, 1.e., intuitively, experimentally, etc. because few 
experimentally-oriented students have the mathematical maturity required* for a mathematical discussion of these concepts.

The previous section pointed out some difficulties present in the teaching of many introductory courses; they are as acute in an introductory statistics course as anywhere, the course at point being no exception. In particular, it experiences some initial problems in student motivation and maintenance of interest. Disciplinewise, it has students from the Plant and Animal Sciences, the Basic Biological Sciences, the Food Sciences, Animal and Human Nutrition, Agricultural Engineering, Rural Sociology, Economics, Child Development, plus a few students from even more diverse areas. Diversity of this extent poses more of a problem with graduate students than with undergraduates because gradua.te students often are single-minded in their disciplinary orientation and consequently are very concerned about immediate relevance to their discipline of the statistical topics under discussion. In fact, for some of them this hang-up is so severe that they cannot grasp a topic unless they can see an example of it within their own disciplinary framework.

Of the many ways to accommodate these difficulties, the development of computer generated laboratory exercises, of demonstration devices and of colorful examples have proved most helpful. Both the students and the instructor have reacted favorably to teaching involving these approaches. Since the objectives of the course have evolved somewhat from year to year and the composition relative to disciplines has been rather variable, it has not been possible to obtain quantitative evaluation of the impact of these approaches on student performance. Speaking subjectively, I do not believe that the level of performance has decreased and probably it has increased. Furthermore, I am confident, from both formal and informal student reaction, that students regard it as a less painful way to learn statistics than had been used previously.

COMPUTER GENERATED EXERCISES were conceived mainly to accommodate the students' diversity of disciplinary interests. It has served this purpose well and consequently has reduced some of the motivational blocks that students have

* A mathematical discussion of these concepts requires a familiarity with mathematics through analysis, i.e., one year more than the usual introductory calculus sequence. 
toward statistics. Further, this approach makes it possible to use the class as an experimental mechanism for intuitively introducing certain topics. This computer use is designed to provide students with experiences that they would not otherwise have; the computer is used for them, they do not use the computer.

Under the present setup, students recelve a set of laboratory exercises (problems to be solved) at the beginning of each week and have a week to work them. Ideally, these problems should illustrate a specific statistical activity in an experimental framework of direct interest to the student. Practically, it is possible to let the student choose to receive problems from a general subjectmatter area such as food science, basic anaimal science, etc. The number of such areas is a matter dictated by availability of facilities. We presently operate with nine. For the sake of in-class discussion, however, everyone should be familiar with the same problems. We have reconciled this by giving the same two problems to everyone and allowing the student's choice of subject-matter interest to dictate the other three. Since some problems are applicable to several areas, we use about twenty problems per week.

Now, how does the computer fit into this process? A substantial computer program has been written to facilitate the individualization of the students' laboratory exercises. The following, which the flowchart can help explain,

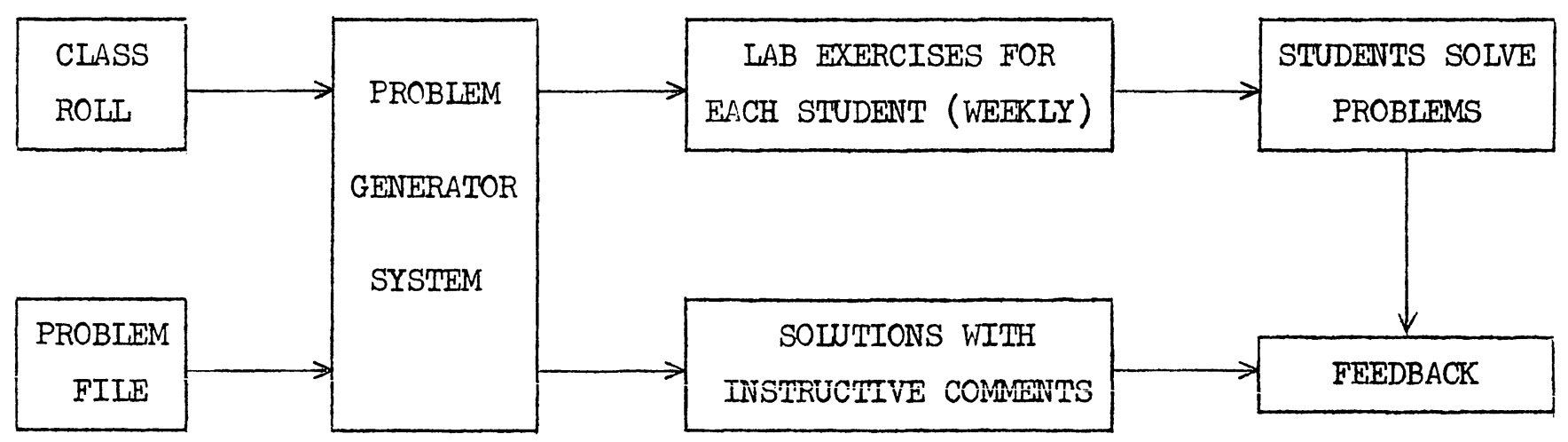

FIOWCHART: COMPUTER GENERATION OF STATISTICS EXERCISES

is an overview of this process: We provide the computer with information about each of the twenty problems, with the allocation of these problems to the subjectmatter areas, and with a list of the students' names and their choice of subject- 
matter areas. It produces a set of exercises for each student. He receives these, works them, and returns them. Simultaneously, the computer produces worked versions of the problems with explanatory comments. After we spot-check the students' work, it and the computer version of the solutions are returned to the student.

The extensiveness of the computer program is dictated by several things. Previously I mentioned the need for providing students with exercises which seem relevant to their disciplinary interests. This requires substantial flexibility in how the same type of problem is presented to the student. A further complication is introduced by the fact that the same set of data can be approached from several directions statistically, depending, of course, on what is sought from the data. The desire to have students practice selection of techniques further complicates the computer program because it must be possible to generate data appropriate for a particular technique at any time during the term. This means that all of our computing must be done in one program rather than in several.

The development of the computer program is only one of the two major development phases. The program must have information about the problems it is to generate. Each problem in the problem file consists of a verbal description, a probabilistic model and questions for the student to answer. The verbal description is a statement of an experimental situation which should make sense to the student. What makes sense to these students? The students themselves should know. Consequently, the first day of class they are given an end-of-term assignment which consists of producing (from a source which is highly relevant to them) two problems of the type we use for exercises. For example, their attention is directed toward their thesis research, the research of a fellow student, a classic example from their discipline, etc. as possible sources of relevant examples. In addition to providing problems for future generations of students, this assignment helps them see the relevance of statistics to their discipline.

The probability model is the vehicle through which the salient features of the original problem are captured without the requirement that the same data always accompany the problem. In fact, the crlginal data is merely one realization of the possible outcomes of the experiment. That is, if the experiment were repeated many times, there would be many results, but their common features can be described in terms of probability. Deviations from this "ideal model" reflect 
only the vagaries present in random sampling, something which is present in any experimental situation. Since many statist1cal concepts can be expressed in terms of probability, the presence of the probabilistic model is very useful. Through it, the concepts can be illustrated in a manner which is not otherwise possible. For example, the sensitivity of a statistical procedure to deviaticns from a specified model depends on the size of the deviation and the amount of empirical information on the deviation, i.e., number of observations. The impact of these two experimental features on the likelihood, or probability, of detecting the deviation can be demonstrated by having, each student consider several computergenerated problems with differing deviations and sample sizes. Then for each problem, they are asked in class how many students detected a deviation between their data and the model. For the problem with no deviation between the generating model and the proposed model, few students detect a difference. This provides an opportunity to emphasize that the vagaries of random sampling will occasionally lead to the conclusion that a difference exists when it does not. More students detect differences between their data and the proposed model as the number of observations or as the size of the deviation increases. Thus their collective experience, summarized in class, serves to help them experience a concept in a nonverbal fashion.

The third part of the problems, the questions to be answered, are framed in subject-matter language. The questions themselves pose little difficulty; the opposite is true of getting the computer to answer every reasonable type of question and of deciding what kind of comments should accompany the numerical results.

The computer, together with the program, generates two sets of output material: a set of problems for each student and solutions to his questions with comments. The computer prints each student's problem set with his name and other information. The introduction of the student's name as part of the heading vas done as part of the individualization. However, this, together with the uniqueness of each student's data, produced an unexpected result. A good many students developed a real sense of pride in their solution of their problems. Students have always compared results; there is no way to prevent it. In fact, it has been encouraged, but now it takes on several new dimensions. Comparison between students with the same problems illustrates the impact of sampling variation on the data and the assoclated conclusions. When problems from different subject- 
matter areas are compared, students see how differing experimental situations lead to the same statistical problem. Further, since each student has a separate random sample of data, student-to-student comparisons of solutions must be at the level of "How should the problem be approached?" rather than at the level of "What is the numertcal answer?". This level of interaction should enhance the learning of concepts.

By now the role of the second set of output material, the answer and comments, should be self-explanatory. However, the comparison of student solutions with those produced by the computer warrants one comment. Graduate teaching assistants presently are responsible for making this comparison. Even before the involvement of the computer, they considered the critical examination of several problems per week from more than 100 students to be a dull chore, an easily understandable attitude. The result was an inefficient feedback process. In particular, undetected errors led students to think that faulty solutions were really correct; when errors were detected, their nature frequently was not made clear. These difficulties were at least secondary reasons for involving the computer; the computer-generated solution sheet provides more complete feedback than was ever possible manually. This makes it possible to now examine one problem seriously for each student and leave him to make the remaining comparisons between his work and the computer solutions.

The present setup leaves one serious problem unsolved. For some students there is a substantial and unavoidable time lag, occasionally as much as a week, between when they complete the problems and when they get feedback. I am beginning to consider how the computer can be used to solve this difficulty. The grading function can be handled rather simply once we get interactive typewriter terminals, but the diagnosis of where a student has gone astray is not a simple problem. However, it will be solved.

DEMONSTRATION DEVICES have been developed to supplement the usual verbal and written presentations of information. They offer a means of intuitively introducing concepts through visual perception and/or involvement in an activity. Since most of the concepts are rather technical, and are built upon preceding concepts, their discussion here is not generally appropriate, but the first device does not suffer these criticisms and is interesting from another viewpoint. Few students appreciate the influence of variation upon experimental outcomes. Indeed, most of 
them are aware of variation within an experiment, but they think of an experiment as having a single outcome. Actually it is one of many possible outcomes, each of which would occur with some unknown probability. In order to broaden their a.titude on this issue, each of them conducts the same, very simple experiment during our first meeting. The experiment consists of determining the number of red corks present in a random sample of five corks which each student draws from a. plastic bottle filled with red and white corks (four red to six white). Some students get no red corks, some get one, and so on, up to a few who even get five. They can hardly fail to notice that they all conducted the same experiment without getting the same results.

This demonstration, suitably executed, has an important secondary effect. It serves to crack the aura of the Professorial image, an act which seems to be particularly essential for those students who are quite apprehensive about the course. This happens because it is impossible to get a dozen or so of these bottles out into the class, the experiments conducted and the outcomes tabulated without some hubbub and excitement, which in turn helps to sink the image of statistics as a hard, cold, humorless discipline. Furthermore, it provides an example which is familiar to the entire class and thus can be a common focal point in subsequent conversations.

COLORFUL EXAMPLES have been developed with primarily motivational concerns in mind. Examples in statistics instruction must either be totally artificial or have some disciplinary flavor. Students rightfully object to more than infrequent use of artificial examples. On the other hand, some students are likely to be unfamiliar with the disciplinary framework of any real example. This difficulty is accentuated if, as is common, an experiment is described in two lines and then several paragraphs are devoted to its statistical analysis and interpretation of the data.

There is a simple solution to this problem. Accompany each example with a series of color slides which can be used to explain the nature of the response, the nature of the experimental concern, and the setup of the experiment itself before the data is introduced. It takes years to develop a complete set of this material, but students' enthusiasm for this type of example is sufficient reward. Again, a fuller discussion of this topic would get into technical concerns and consequently will not be pursued here. 


\section{ACKNOWLEDGMENT}

This paper was prepared while I was a participant in Project Changeover, 1968, a workshop on innovation in higher education funded by the Kettering Foundation and administered by the Union for Research and Experimentation in Higher Education. This and the previous summer's workshop certainly made me more sensitive to a number of the issues discussed above. For this I am grateful. 